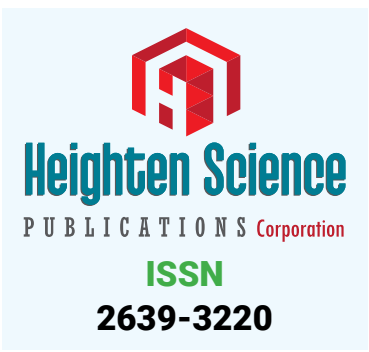

*Address for Correspondence: Long Ching, Department of Neurosurgery, Queen Elizabeth Hospital, Hong Kong, Tel: (+852) 97504477; Email: chinglong1994@hotmail.com

Submitted: 27 June 2019

Approved: 08 July 2019

Published: 09 July 2019

Copyright: (c) 2019 Ching L, et al. This is an open access article distributed under the Creative Commons Attribution License, which permits unrestricted use, distribution, and reproduction in any medium, provided the original work is properly cited

D) Check for updates
Case Report

\section{Mimicking multiple sclerosis - Ghost tumor that comes and goes in different parts of the brain without any treatment}

\author{
Long Ching ${ }^{1 *}$, Ming Him Yuen1, Tak Lap Poon', Fung Ching \\ Cheung ${ }^{1}$, Shun Hin Ting ${ }^{2}$ and Wing Chi Fong ${ }^{3}$ \\ 'Department of Neurosurgery, Queen Elizabeth Hospital, Hong Kong \\ 2Department of Pathology, Queen Elizabeth Hospital, Hong Kong \\ ${ }^{3}$ Department of Medicine, Queen Elizabeth Hospital, Hong Kong
}

\section{Summary}

Lesions that spontaneously come and go in central nervous system without any treatment at different time points and at different locations (CNS) usually lead ones to think of the possibilities of multiple sclerosis. However, sometimes there are exceptions. Surgical biopsy remains an important tool for definitive diagnosis in difficult cases. We report a case of intracranial diffuse large B cell lymphoma that spontaneously disappeared without any treatment and then reappeared at different time points and different locations.

\section{Introduction}

Lesions that disappear spontaneously without treatment then reappear at different locations in the CNS at different time points in imaging are one of the classical radiological features of multiple sclerosis. On the other hand, lymphomas involving CNS are known to be steroid sensitive. They can disappear completely after steroid treatment and thus are sometimes referred as ghost tumours. Despite the name of ghost tumours, lymphomas in the CNS resolving spontaneously without any treatment is still unheard of in the literature. We report a case that had intracranial lesions disappear spontaneously without any treatment then reappear at different locations. The histological diagnosis confirmed to be diffuse large B cell lymphoma.

\section{Case Report}

57-year-old lady with the history of diffuse large B cell lymphoma which was successfully induced remission by R-CHOP regimen in 2012, presented with dizziness and blurring of vision in June 2017. Private PET-CT was negative, while MRI brain showed an abnormal enhancing nodular lesion that showed restricted diffusion over right frontal periventricular region (Figure 1). Bone marrow aspiration showed no malignant cells [1]. Lumbar puncture was unremarkable in biochemical investigation, oligoclonal band, cytology and culture. Lymph node biopsy could only showed lymphoproliferative lesion. In view of inconclusive findings (Table 1) and absence of definite diagnosis, follow up imaging was arranged and no steroid or chemotherapy was given.

MRI brain was repeated in September 2017. The known right frontal periventricular 


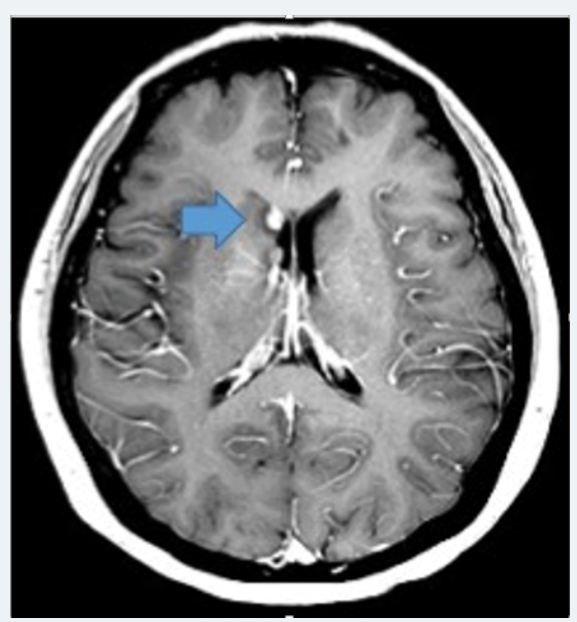

Figure 1: T1 axial cut of MRI brain in June 2017. There was a contrast enhancing lesion in the right periventricular region (Blue arrow)

\begin{tabular}{|c|c|c|}
\hline & Location of lesion & Action taken \\
\hline June 2017 & $\begin{array}{l}\text { right frontal periventricular } \\
\text { region0 }\end{array}$ & $\begin{array}{l}\text { - Bone marrow aspiration: no malignant cells seen } \\
\text { Lumbar puncture: negative in oligoclonal, cytology and } \\
\text { culture. Normal biochemical investigation } \\
\text { - Cervical Lymph Node cytology: Lymphoproliferative lesion }\end{array}$ \\
\hline $\begin{array}{l}\text { September } \\
\quad 2017\end{array}$ & $\begin{array}{c}\text { Left periventricular region near } \\
\text { caudate head }\end{array}$ & Biopsy offered: refused by patient \\
\hline July 2018 & Left parietal lobe & Autoimmune markers ${ }^{1}$ : All negative \\
\hline
\end{tabular}

'Autoimmune markers: Include NMDA-R Ab CBA, S Neuronal (V-G), K+Channel Ab, S, LGI1-IgG CBA, S, CASPR2-IgG CBA,S, GAD65 Ab Assay, S, GABA-B-R Ab CBA, S, AMPA-R Ab CBA, S, ANNA-1, S, Reflex Added, ANNA-2, S, ANNA-3, S, AGNA-1, S, PCA-1, S, PCA-2, S, PCA-Tr, S, Amphiphysin Ab, S, N-Type Calcium Channel Ab, P/Q-Type Calcium Channel Ab, AChR Ganglionic Neuronal Ab, S, CRMP-5-IgG,S.

lesion disappeared while there was a new T2-weighed hyperintense lesion at the left caudate head (Figure 2), with contrast enhancement in T1 and restricted diffusion. Surgical biopsy was offered but was refused by the patient. Another MRI brain scan was repeated in July 2018 which showed a new left parietal lesion (Figure 3), while other lesions disappeared [2,3]. Autoimmune markers for autoimmune encephalitis were negative. Differential diagnosis including multiple sclerosis and autoimmune encephalitis were then ranked less likely. The patient developed transient dysphasia in Aug 2018, subsequent CT brain showed the known left parietal lesion with new perilesional edema. Surgical biopsy was offered and then agreed. Craniotomy for open biopsy was done in September 2018. The cortical surface was apparently normal looking but intraoperative ultrasound showed hyperechoic signals. Further exploration of the parietal region found greyish tissue. Biopsy was taken in that area and frozen section (Figure $4 \mathrm{a}$ and $4 \mathrm{~b}$ ) showed cells with round nuclei, nuclear membrane irregularity, open chromatin with nucleoli, scant cytoplasm and 'foamy cells' which are typical lymphoid features, and final pathology was proven to be diffuse large B cell lymphoma. The patient was then taken care by hematologists for chemotherapy.

\section{Discussion}

Diffuse large B cell lymphoma (DLBCL) is the most common histologic subtype of non-Hodgkin lymphoma (NHL) which accounts for approximately 25 percent of NHL cases. The incidence of DLBCL is approximately 7 cases per 100,000 persons per year in the United States and England. According to Hong Kong Cancer Registry, lymphoma accounts for $3 \%$ of total incidence of cancer in the year of 2016. Central nervous system involvement by DLBCL is not common at presentation and will occur at some points during the disease course in less than 10 percent of patients [4,5]. Lymphomas involving Central Nervous System are well known to be steroid sensitive. Initial response rate of 


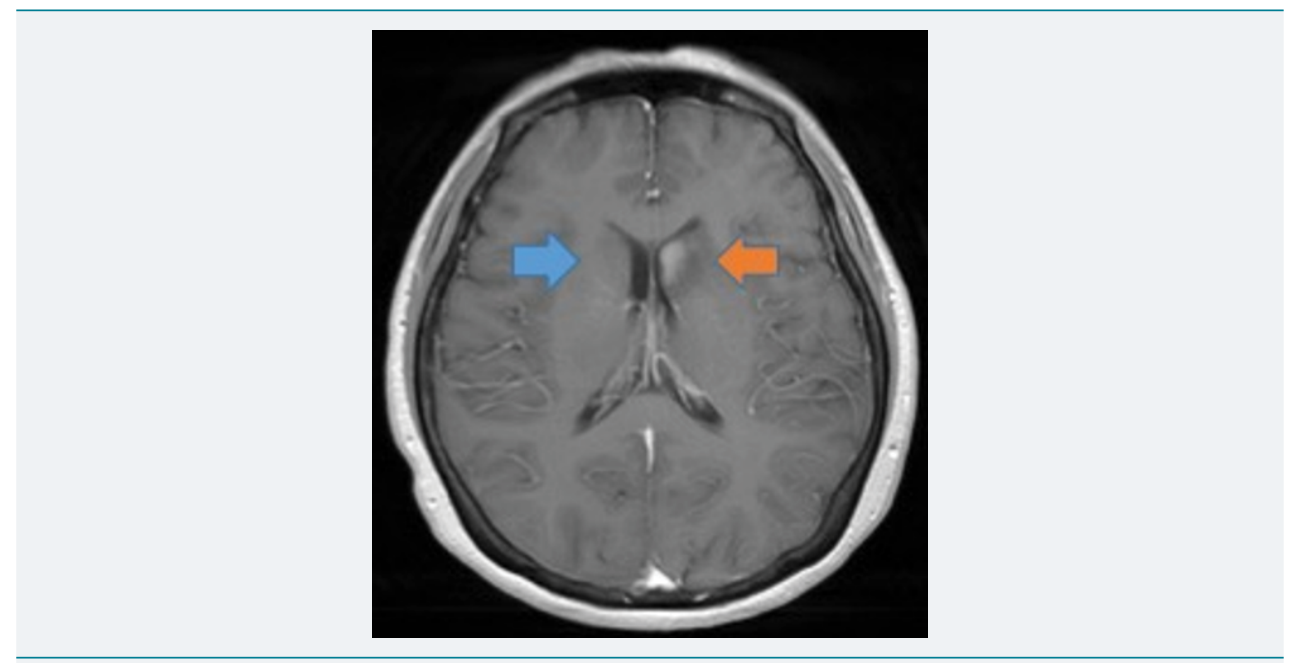

Figure 2: T1 axial cut of MRI brain in September 2017. The old lesion disappeared (blue arrow) and a new lesion in the left caudate head appeared. (Orange arrow).

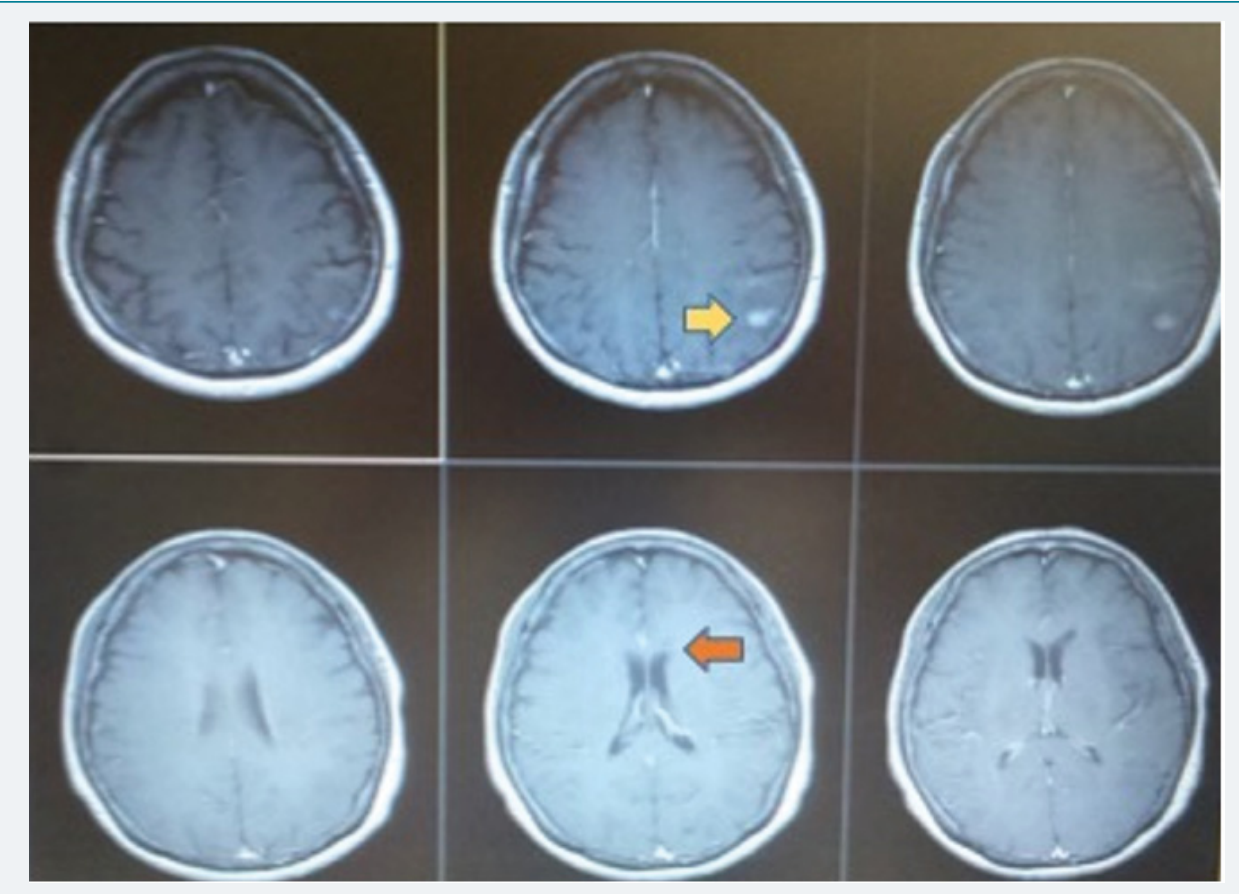

Figure 3: T1 axial cut of MRI brain in July 2018. The old lesion (orange arrow) disappeared and a new lesion in left parietal region appeared (yellow arrow)

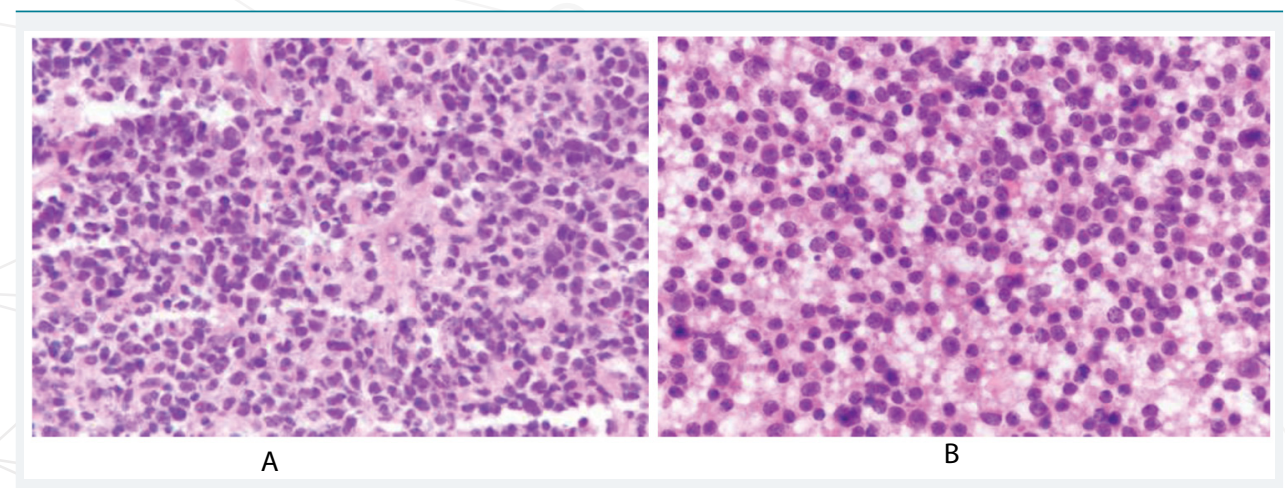

Figure 4: a: Frozen section of deeper part showing cells with obvious cytologic atypia which point to the malignant nature. The high nuclear-to-cytoplasmic ratio, open chromatin, multiple nucleoli and overall round nuclear outline are suggestive of lymphoma. b: Frozen section of the same site showing nuclear membrane irregularity, open chromatin with nucleoli and scant cytoplasm suggests lymphoid origin. Foamy macrophages are also present. 
Central Nervous System lymphoma can be as high as 70 percent. Therefore, sometimes they are referred as 'ghost tumours'. However, lesions that disappear spontaneously without treatment then reappear at different locations in the central nervous system at different time points in imaging are one of the classical features of multiple sclerosis. Multiple sclerosis is characterized radiologically by one or more T2-hyperintense lesions in at least two of the four MS-typical regions of the Central Nervous System including periventricular, cortical, infratentorial and spinal cord. Therefore, multiple sclerosis was initially suspected in this case. Underlying pathophysiology of ghost tumor phenomenon is still unclear. Foamy cells are occasionally found in ghost tumor specimen in literature. In this case, foamy cells are present which may signifies the roles of macrophages in this phenomenon and the detailed pathophysiology has to be further investigated. From this case, it can be well illustrated that lymphoma can come and go at different locations in Central Nervous System without any treatment. Surgical biopsy remains an important tool to establish the definitive diagnosis in difficult cases like this one.

\section{Author Contributions}

All authors contributed to the design, acquisition and interpretation of data, drafting of the article, and critical revision for important intellectual content.

\section{References}

1. Hoffman S, Propp J, Mccarthy B. Temporal trends in incidence of primary brain tumors in the United States, 1985- 1999. Neuro Oncol. 2006; 8: 27-37. PubMed: https://bit.ly/2Jg6Qz6

2. Ferracini R. Primary malignant non-Hodgkin's lymphomas of the central nervous system in immunocompetent patients: Diagnostic, prognostic and therapeutic criteria. Pathologica. 1997; 89: 146-154. PubMed: https://bit.ly/2XtWgZc

3. Takekawa $\mathrm{H}$, Hozumi Hirata, Yamazaki. A spontaneously vanishing primary cerebral lymphoma "ghost tumour". J Neurol Neurosurg Psychiatry. 2008; 79: 1159. PubMed: https://bit.ly/2XsME6b

4. Naeem S, Niazi F, Baig A, Sadiq H, Sattar M. Primary CNS Lymphoma vs. Tumefactive Multiple Sclerosis: A Diagnostic Challenge. J Coll Physicians Surg Pak. 2018; 28: 66-68. PubMed: https://bit.ly/30gie3K

5. Ryotaro I, Yuko S, Satoru S, Kazuo K. CSF and clinical data are useful in differentiating CNS inflammatory demyelinating disease from CNS lymphoma. Mult Scler. 2018; 24: 1212-1223. PubMed: https://bit.ly/2Jtu8QW 